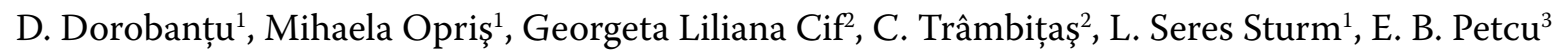

\title{
Basal Cell Carcinoma Aggressiveness, Molecular Factors And Therapy: A Clinician Perspective
}

${ }^{1}$ University of Medicine and Pharmacy, Târgu Mureş

${ }^{2}$ Mures County Hospital, Târgu Mureş

${ }^{3}$ University of Medicine and Pharmacy Târgu Mureș, Griffith University School of Medicine

\begin{abstract}
.
Basal cell carcinoma represents the most common skin and epithelial cancer. Most of the patients are cured by surgery. However, some cases display fullblown aggressiveness which has a dual connotation. The tumour may reach an impressive size being locally destructive while in rare cases, basal cell carcinomas may metastasize. If this agressivetumour is located on the face or neck, the surgeon is confronted with a clinical dilemma since total removal of the lesion with clear margins is impossible. Therefore, nonsurgical approaches need to be adopted in these cases. A better understand of the molecular pathways could theoretically lead to new improved therapeutic treatments. The current paper presents an update on the molecular factors with clinical importance for the treatment of basal cell carcinoma in parallel with presentation of an aggressive case as well as a review of the current therapeutic methods.
\end{abstract}

Key words: BCC, metastatic basal cell carcinoma, Imiquimod, Tazarotene, 5-Fluorouracil, cryotherapy

Eugen Bogdan Petcu MD, PhD

University of Pharmacy and Medicine Tg. Mures

$38 \mathrm{Gh}$. Marinescu, Tg. Mureş,

Tel: +40-265-215-551

E-mail: e.petcu@griffith.edu.au

\section{Introduction}

It is known that basal cell carcinoma of skin is a malignant tumour made up of neoplastic lobes, trabeculae, strips and cords of basal cells. Remarkably, it is the most common skin cancer representing 30\% of all cutaneous cancers and $60-80 \%$ of epithelial skin cancers [1].

Basal cell carcinoma is a tumor commonly observed in people with light skin (Caucasians) who live low altitude regions and are at risk of sun burns.It occurs predominantly on sun-exposed skin.In Australia, there are 2000 cases of basal cell carcinomas per 100000people [2]. The incidence increases in elderly, but it is also described in young patientsand children displaying the nevoid basal cell epithelioma syndrome [3], [4].Basal cell carcinoma is seen twice as frequent on the cephalic extremity compared with other locations such as trunk, upper limb and lower limbs in which case the incidence is much lower. In addition, some cases are cured by simple surgical resection while others are aggressive.

\section{Aggressive basal cell carcinoma}

In most cases of basal cell carcinoma, the natural evolution is slow and the prognosis is good compared to other types of skin malignancies. Most of the patients have a high cure rate. We believe that the term "aggressive" which is applied in some case has a dual connotation.In some circumstance this may describe a local "aggressiveness" while in some other 
cases this includes the process of metastasis.

Local "aggressiveness" is usually characterized by extensive growth leading to significant tissue destruction [5]. If neglected the initial tumor can reach a significant size with involvement of the nearby structures. In addition, the location of the lesion especially on the face may preclude a total excision of the tumor. These cases may also be regarded as locally aggressive (fig 1, fig 2).

As shown above, the neoplastic growth may be significant involving large parts of the face. In these cases, it is not uncommon that residual tumor cannot be completely excised or the surgical margins show residual temporal foci. A subsequent intervention with curative intent may be very difficult and other therapeutic methods should be offered to these patients.

In addition, basal cell carcinoma especially the morpheiform type has a tendency to metastasize Natural evolution of these cases is characterized by numerous recurrences with brief periods of apparent clinical cure [6]. Although, the incidence of dissemination is generally below $0.1 \%$, cases of metastases in the lymph nodes, lungs, bones, liver have been reported [5].According to Walling et al (2004) apart from metastasis an aggressive behavior for a BCC is associated with deep invasion and local recurrence. However, the authors suggest the following factors as definite markers of

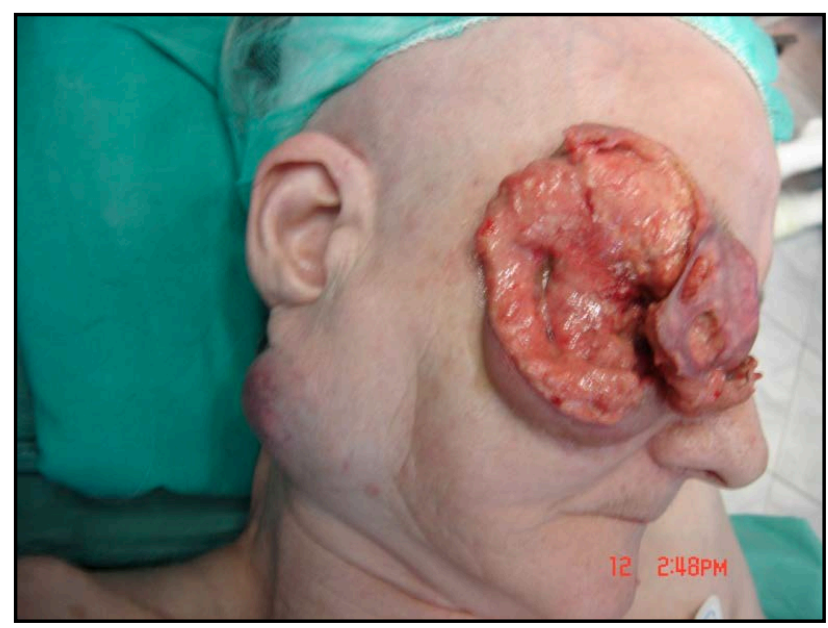

Figure 1 - Basal cell carcinoma: locally infiltrating, ulcerated involving the right periorbital, orbital, and zygomatic areas. A secondary growth is noted at the mandibular angle aggressiveness: tumor size, duration of development, histology and perineural spread [7].

\section{Metastatic basal cell carcinoma: Case presentation}

An 83 year-oldfemale, presented with a large ulcerated tumour $(10 \mathrm{~mm} \times 8 \mathrm{~mm})$ involving the right orbital and periorbital area, the ocular globe being macroscopically involved by the neoplastic growth (fig 3).

Interestingly, the radiological evaluation revealed an asymptomatic left atrial myxoma which was asymptomatic. In Gorlin's syndrome known as the nevoid basal cell carcinoma syndrome, the patients present at a young age with cardiac fibromas and loss of PTCH1 gene locus [8]. The association between a basal cell carcinoma and a cardiac myxoma has not been previously described in the literature. However, in order to define a new genodermatosis more cases need to be identified displaying these two types of tumors. Therefore, we have considered these two lesions as being synchronous.

After counseling, tumor resection was performed along with right ocular globe enucleation. The surgeon performed a tissue sparing resection with clear margins (Fig 4). The resection avoided major vascular and nervous branches located in the

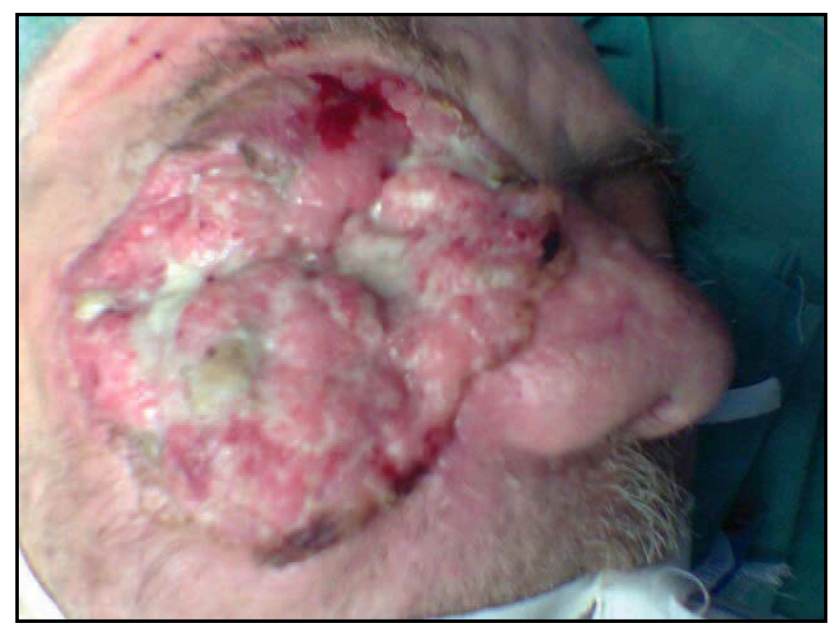

Figure 2 - Basal cell carcinoma: locally infiltrating and ulcerated covered by purulent exudates involving the right periorbital, orbital and zygomatic areas, invading the nasal pyramid 


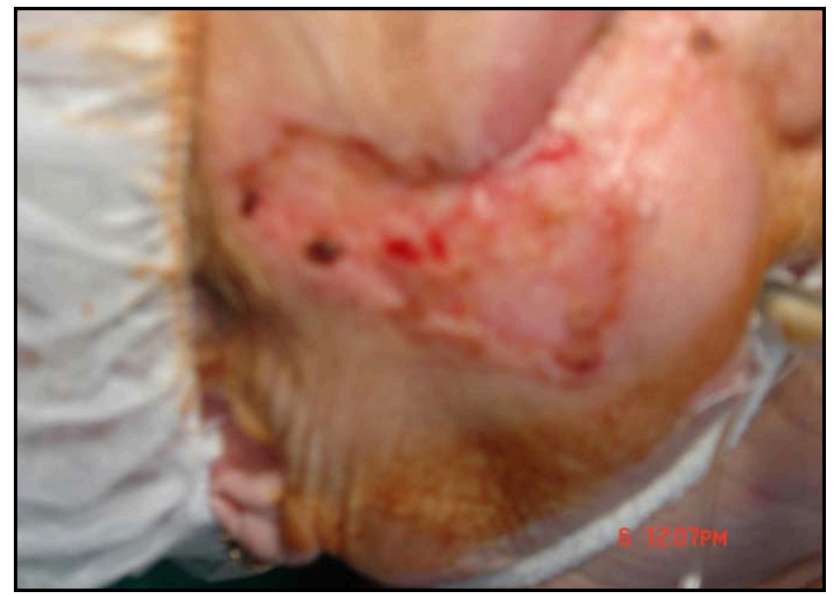

Figure 3 - Basal cell carcinoma ulcerated invading the right periorbital and orbital areas

area of interest. Although some superficial nervous and vascular branches might have been damaged during the actual resection, the actual amount of tissue removed did not interfere significantly with subsequent wound healing (fig 5).

The second stage of the surgical intervention included facial reconstruction with free split skin graft taken from the ipsilateral thigh (fig 6).

The surgical wound healed without complications with a complete functional scar at 14 days (fig 7, fig 8). However, since the patient presented for diagnosis and treatment very late, the general surgical outcome although curative for basal cell carcinoma may be regarded as invaliding especially considering the enucleation of the right orbital globe which could not be avoided.

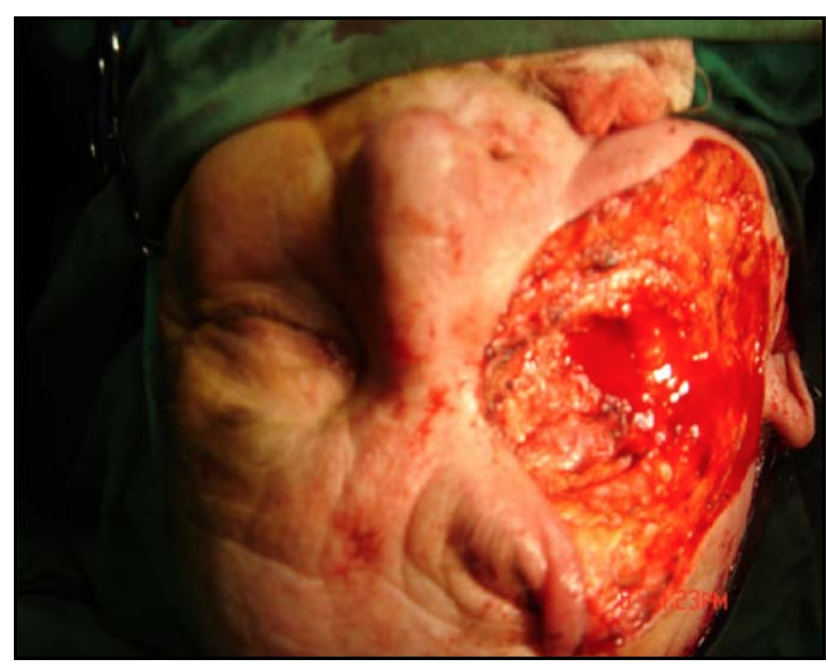

Figure 4 - Intra-operative aspect

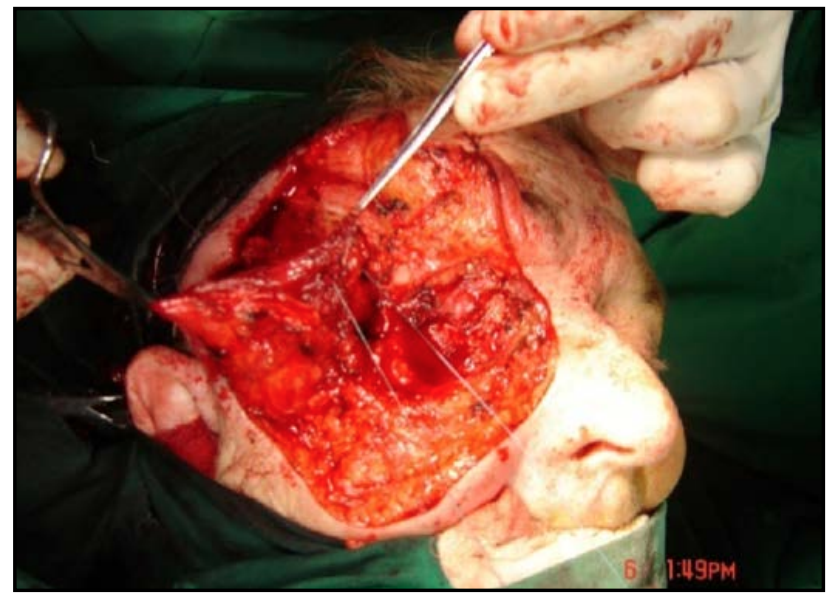

Figure 5 - Intraoperative aspect showing preservation of muscles and associated vessels and nerves

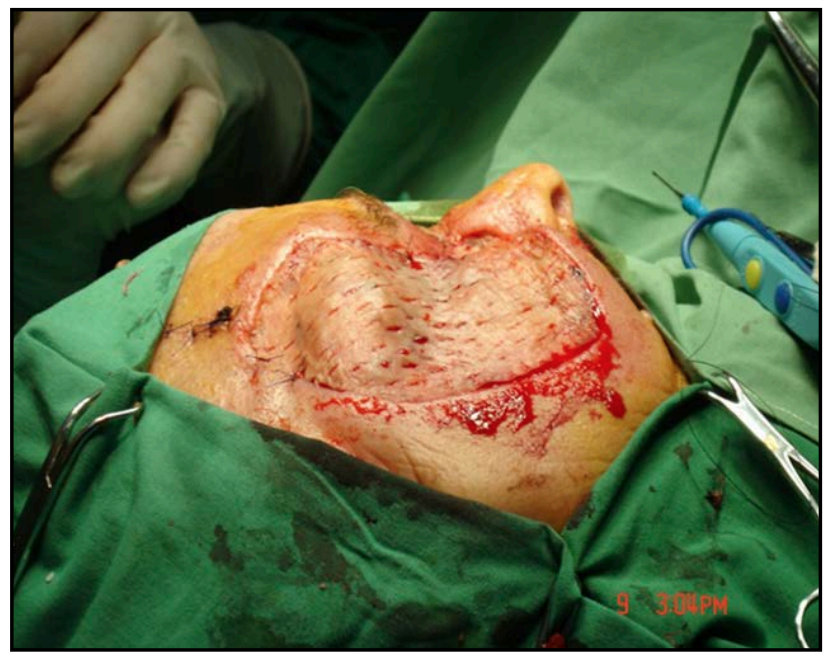

Figure 6 - Intraoperative aspect: free split skin graft

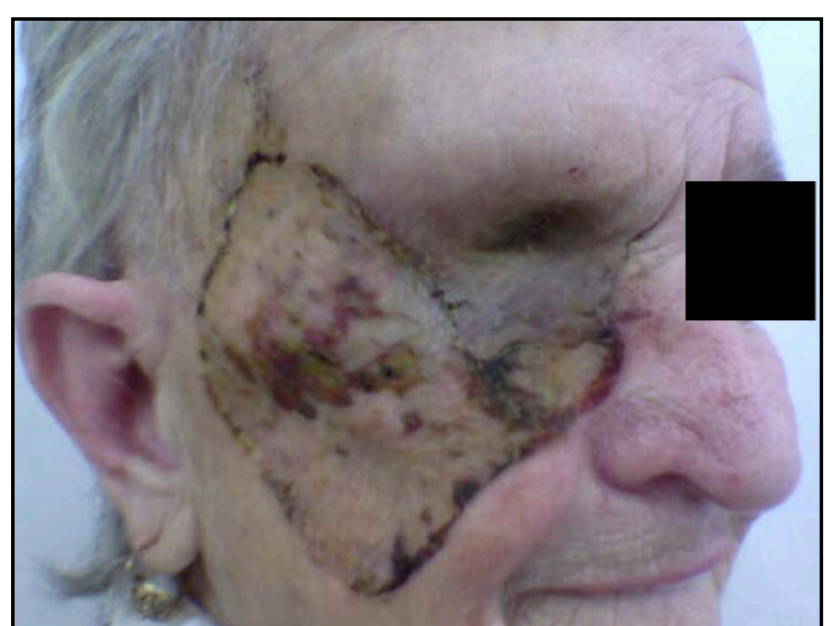

Figure 7 - Patient at $14^{\text {th }}$ day post-surgery: antero-lateral view 


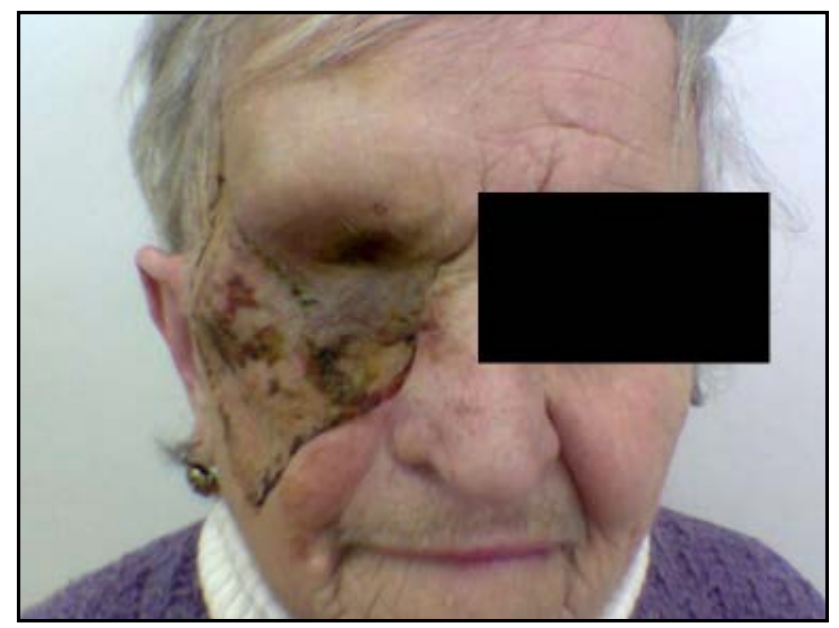

Figure 8 - Patient at $14^{\text {th }}$ day post-surgery: anterior view

\section{Discussion}

\section{New molecular insights related to basal cell carcinoma}

Basal cell carcinoma represents a uni- or multifocal process. However, it determines only rarely metastasis. There are less than 300 metastatic cases described in the literature of all times.Metastatic basal cell carcinoma is defined as primary cutaneous basal cell carcinoma that spreads to distant sites and determines histologically similar deposits of BCC. Some histological types (morpheic, infiltrating, basosquamous) indicate an increased risk of metastasis. Also, the presence of perineural invasion indicates a propensity to metastatic behavior $[9$, 10]. Recent studies published by Young et al (2008) have revealed that compared with squamous cell carcinoma, basal cell carcinoma is characterized by lower levels of DNA mismatched repair proteins [11]. The authors suggest that this difference determines the net aggressiveness of squamous cell carcinomas compared with basal cell phenotype. However we do not know if there are any differences at the DNA mismatched repair proteins between metastatic basal cell carcinomas and non-metastatic types and further studies are needed in this field.

As shown above it may be locally destructive, especially if neglected for a long time.In these cases, regardless the histological type, the initial lesions are locally infiltrating, invading muscles and bone.Recent studies have tried to explain this behavior by changes in the gene expression.Abnormalities were recorded in genes modulating the functionality of extracellular matrix and cell junctions, motility, metastasis, oncogenes, tumor suppressors, DNA repair, cell cycle, immune regulation and angiogenesis. Most of these are also involved in the metastatic process. The net result of these abnormal genetic profiles is represented by a massive local destruction and in some cases of course, metastatic growth. Most importantly, all the above mentioned genes represent a potential therapeutic target [12].

It is known that the basal cell carcinoma displays several histopathological patterns such as superficial, nodular (pigmented, keratotic), micronodular, infiltrative, morpheiform. One may argue that the variety of histopathological forms might be determined by various pathways activated in this malignancy.Zhang $M$ et al (2012) report in a genome-wide association study of basal cell carcinoma of skin several new pathways involved in the BCC carcinogenicity: the heparan sulfate biosynthesis pathway, the mCalpain pathway, the Rho cell motility signaling pathway and the nitric oxide pathway [12]. These add up to the previous studies that have revealed factors and pathways such as the hedgehog pathway, p53 and PTCH who may have a crucial role in carcinogenesisof various forms of basal cell carcinoma $[14,15,16]$.

\section{Current therapeutic concepts: sneak-peak}

\section{Surgical therapy}

The therapeutic choice depends on the histological type and size of the tumour, stage and local facilities of the institution. The most effective treatment is still considered to be surgical excision which aims to completely remove the tumor tissue leaving clear margins. Since basal cell carcinoma occurs most frequently in sun-exposed areas such as head and neck, one of the most important therapeutic principle is to save as much as possible viable tissue to allow the best functional and esthetic results. However, the currently recommended lateral excision margin for basal cell carcinoma is 4 millimeters, 
although this may not be possible in all cases [17]. Interestingly, a new multicenter study conducted in several EU countries (EPIDERM: European Prevention Initiative for Dermatological Malignancies) revealed that tumors of the head including $\mathrm{BCC}$ were more likely to be treated by non-surgical methods than those at other locations. The same authors report that at the present time, cryotherapy is the most widely used nonsurgical therapeutic option before pharmacological agents such as imiquimod or photodynamic therapy. The last therapeutic preferences are represented by 5-fluorouraciland diclofenac with hyaluronic acid [18].

\section{Nonsurgical methods}

\section{Cryotherapy}

Cryotherapy is a physical treatment promoting tumour cell destruction. It seems to be the preferred method in cases characterized by the presence of basal cell carcinomas on the face which are not easy amenable to surgery [18]. A very recent study conducted in patients refractory to imiquimod treatment revealed a complete clinical response in most cases $(80 \%)$. Only a single unresponsive case was recorded in 23 patients [19].

Radiation therapy

A recent study published by Olschewski et al (2006) highlight the role of radiotherapy in the treatment of basal cell carcinoma of the face and head. According to their data the authors report cure rates of $92-96 \%$ after radiotherapy. The dosage schedule was $5 \times 3$ Gray/week up to a total dose of 57 Gray in $95 \%$ of patients. Remarkably no residual disease or recurrence was observed. In nearly most of the patients the esthetic result was excellent or good. The authors conclude that low grade radiotherapy might be the gold standard from BCC located on the face or neck [20]. In addition, Mendenhall et al (2006) suggest that radiotherapy is very useful in aggressive tumours after surgery especially when clear margins cannot be obtained with confidence [21].However, the shortcomings of this treatment are represented by the high-cost of the equipments and the compulsory assistance from highly specialized medical and scientific personnel. Last but not least, it already widely accepted that high doses of ionizing radiation therapy may promote skin cancer including basal cell carcinoma.

\section{Photodynamic therapy}

The principle behind this method is represented by the wavelength stimulation of previously administered porphyrins which ultimately promotes synthesis of oxygen radicals which subsequently destroy the neoplastic tissue.Recent research suggest that although it needs further evaluation, photodynamic therapy using topical porphyrin-precursors may be an option in selected cases in which the lesion is located superficially [22]. The results of a 5-year follow-up study with methyl aminolevulinate acid in patients with BCC located on the eyelid revealed that photodynamic therapy is an efficient method, especially in cases in which surgical excision is very difficult to be performed with clear margins [23].

\section{Pharmacologicaltreatment}

Topical 5-fluorouracil 5\%

5-Fluorouracil blocks the methylation of deoxyuridylic acid and thymidylatesynthetase by interfering with DNA synthesis. The final result is represented by inhibition of cell proliferation. This agent approved by the US Food and Drug Administration has been used for many years to treat actinic keratosis and superficial basal cell carcinoma [24]. In an open-label, randomized study of 122 patients with BCC confirmed histologically, regardless of the dosage regimen, $91 \%$ of the patients had complete resolution of the tumours. This suggests that topical 5-Fluorouracil may be an effective alternative to surgery in selected cases [25].

Imiquimod

Drehs et al (2002) reported that Imiquimod $5 \%$ cream (Aldara) is effective in treating superficial BCC unresponsive to other therapies [26]. Imiquimod is approved by the US Food and Drug Administration for treating superficial basal cell carcinoma and is currently administered in cases presenting with multiple or large superficial BCC especially if the lesions are located on critical zones of the face [27].A recent study has revealed that Imiquimod 5\% cream is very effective in shrinking the tumour in cases of 
nodular basal cell carcinoma before Moh's surgery. This protocol promotes a significantly reduced defect size [28].

\section{Tazarotene}

Tazarotene is a retinoid agent with retinoic acid receptor (RAR) beta/gamma specificity which has revealed a significant anti-tumoural action in selected cases of basal cell carcinoma. The actions of Tazarotene seem to be mediated via RAR gamma activation [29]. Tazarotene $0.1 \%$ gel was administered daily over 6 months to 154 small superficial and nodular BBCs. At the end of the study, $70.8 \%$ of cases revealed more than $50 \%$ clinical and dermoscopic reduction in size. In addition more than $30 \%$ of cases were disease-free at 3 years after tazarotene administration suggesting that this pharmacological alternative may be the treatment of choice in some cases. Remarkably, the unresponsive cases were characterized by increased levels of p53 and cellular retinol binding protein-1. Histologically, these cases were described as keratotic BCC [30].

In conclusion, although most of the basal cell carcinomas have a favorable outcome, some forms are aggressive. This behavior may be related to the local destruction or the metastatic dissemination. The chances of a total cure in these cases are reduced and may be determined by the stage of presentation as well as molecular and genetic abnormalities which may be present.

At the present time basal cell carcinoma may be treated by a variety of local and systemic approaches prevention is of paramount importance. Sun exposure between the hours of highest UV activity and sunbathing should be avoided. Sun protection creams and clothing should be used at all times especially by those living in warm climates. Exposure to arsenic and ionizing radiation should be avoided as well. Those known with certain genetic abnormalities such as Gorlin syndrome should be very closely followed-up as well as those with aggressive forms of basal cell carcinoma.Last but not least counseling and psychological support should be provided to all patients and especially to those with aggressive lesions on the face.

This paper is partially supported by the Sectoral Operational Programme Human Resources
Development, financed from the European Social Fund and by the Romanian Government under the contract number POSDRU/89/1.5/S/60782

\section{References}

1. Dimitrescu Al.,Trifu P. (1992) Precancerele Si Cancerele Cutanate Vol I Pag 9-26,149-185., Ed. Medicala, Bucuresti.

2. Green A, Battistutad,Hart V .S (1996). Cancer In A Subtropical Australian Population. Thenambour Study Group. Am J Epidemiol; 144:1034-1040.

3. Morarul (1980). Anatomiepatologică vol II. Ed. Medicala, Bucuresti.

4. Rahbari H, Mehregan Ah. (1982) Basal Cell Epithelioma (Carcinoma) In Children And Teenagers. Cancer ;49:350-363.

5. Ji R C. (2006) Lymphatic Endothelial Cells, Lymphangiogenesis, And Extracellular Matrix. Lymphat Res Biol. ;4(2):83-100.

6. Cruse P.J.E., Mcphedran N.T. (1987) Wound Healing Andmanagement. In:,Sabiston's Textbook Of Surgery", Editia A 14-A. W. B. Saunders Company, Philadelphia : 117-121.

7. Walling $\mathrm{Hw}$, Fosko $\mathrm{Sw}$, Geraminejad $\mathrm{Pa}$, Whitaker Dc, Arpey Cj. (2004) Aggressive Basal Cell Carcinoma: Presentation, Pathogenesis, And Management. Cancer Metastasis Rev. ;23(34):389-402.

8. Scanlan D, Radio Sj, Nelson M, Zhou M, Streblow R, Prasad V, Reyes C, Perry D, Fletcher S, Bridge Ja. (2008) Loss Of The Ptch1 Gene Locus In Cardiac Fibroma.Cardiovascpathol.17(2):93-7.

9. Weiss Gj, Korn Rl. (2012) Metastatic Basal Cell Carcinoma In The Era Of Hedgehog Signaling Pathway Inhibitors.Cancer.

10. Ting Pt, Kasper R, Arlette Jp. (2005)Metastatic Basal Cell Carcinoma: Report Of Two Cases And Literature Review. J Cutan Med Surg. (1):10-5.

11. Young Lc, Listgarten J, Trotter Mj, Andrew Se, Tron Va. (2008) Evidence That Dysregulated 
Dna Mismatch Repair Characterizes Human Nonmelanoma Skin Cancer. $\mathrm{Br} J$ Dermatol. 158(1):59-69.

12. Howell Bg, Solish $\mathrm{N}$, Lu C, Watanabe $\mathrm{H}$, Mamelak Aj, Freed I, Wang B, Sauder Dn. (2005) Microarray Profiles Of Human Basal Cell Carcinoma: Insights Into Tumor Growth And Behavior. J Dermatol Sci.39(1):39-51. Epub 2005 Apr 18.

13. Zhang M, Liang L, Morar N, Dixon Al, Lathrop Gm, Ding J, Moffatt Mf, Cookson Wo, Kraft P, Qureshi Aa, Han J. (2012) Integrating Pathway Analysis And Genetics Of Gene Expression For Genome-Wide Association Study Of Basal Cell Carcinoma. Hum Genet.;131(4):615-23.

14. Bale Ae, Yu Kp. (2001) The Hedgehog Pathway And Basal Cell Carcinomas. Hum Mol Genet. 10(7):757-62.

15. Wicking C, Mcglinn E. (2001) The Role Of Hedgehog Signalling In Tumorigenesis.Cancer Lett.173(1):1-7.

16. Zhang H, Ping Xl, Lee Pk, Wu Xl, Yao Yj, Zhang Mj, Et Al.(2001) Role Of Ptch And P53 Genes In Early-Onset Basal Cell Carcinoma. Am J Pathol. 158(2):381-5.

17. Weinstein Mc, Brodell Rt, Bordeaux J, Honda K. (2012) The Art And Science Of Surgical Margins For The Dermatopathologist.Am $J$ Dermatopathol. 34(7):737-45.

18. Ferrandiz L, Ruiz-De-Casas A, Trakatelli M, De Vries E, Ulrich M, Aquilina S, Saksela O, Majewski S, Ranki A, Proby C, Magnoni C, Pitkänen S, Kalokasidis K, Siskou S, Hinrichs B, Altsitsiadis E, Stockfleth E, Moreno-Ramirez D; Epiderm Group. (2012) Assessing Physicians' Preferences On Skin Cancer Treatment In Europe.Br J Dermatol. 67suppl 2:29-35.

19. Messeguer F, Serra-Guillen C, Echeverria B, Requena C, Sanmartin O, Llombart B, Guillen C, Nagore E. (2012) A Pilot Study Of Clinical Efficacy Of Imiquimod And Cryotherapy For The Treatment Of Basal Cell Carcinoma With Incomplete Response To Imiquimod. $J$ Euracaddermatolvenereol. ;26(7):879-81.

20. Olschewski T, Bajor $\mathrm{K}$, Lang B, Lang E, Seegenschmiedt Mh. (2006) Radiotherapy Of Basal Cell Carcinoma Of The Face And Head:
Importance Of Low Dose Per Fraction On LongTerm Outcome. J Dtschdermatolges. 4(2):12430.

21. Mendenhall Wm, Amdur Rj, Hinerman Rw, Cognetta Ab, Mendenhall Np. (2009) Radiotherapy For Cutaneous Squamous And Basal Cell Carcinomas Of The Head And Neck. Laryngoscope. 119(10):1994-9

22. De Vijlder Hc, Sterenborg $\mathrm{Hj}$, Neumann Ha, Robinson Dj, De Haas Er. (2012) Light Fractionation Significantly Improves The Response Of Superficial Basal Cell Carcinoma To Aminolaevulinic Acid Photodynamic Therapy: Five-Year Follow-Up of A Randomized, Prospective Trial. Acta Derm Venereol. 17;92(6):641-7.

23. Puccioni M, Santoro N, Giansanti F, Ucci F, Rossi R, Lotti T, Et Al. (2009) Photodynamic Therapy Using Methyl Aminolevulinate Acid In Eyelid Basal Cell Carcinoma: A 5-Year Follow-Up Study. Ophthalplastreconstr Surg.;25(2):115-8

24. Moore Ay. (2009) Clinical Applications For Topical 5-Fluorouracil In The Treatment Of Dermatological Disorders.J Dermatolog Treat. 20(6):328-35.

25. Miller Bh, Shavin Js, Cognetta A, Taylor Rj, Salasche S, Korey A, Et Al. (1997) Nonsurgical Treatment Of Basal Cell Carcinomas With Intralesional 5-Fluorouracil/Epinephrine Injectable Gel. J Am Acad Dermatol.36(1):72-7

26. Drehs Mm, Cook-Bolden F, Tanzi El, Weinberg Jm. (2002) Successful Treatment Of Multiple Superficial Basal Cell Carcinomas With Topical Imiquimod: Case Report And Review Of The Literature. Dermatol Surg. 28(5):427-9

27. Tandon Y, Brodell Rt. (2012) Local Reactions To Imiquimod In The Treatment Of Basal Cell Carcinoma. Dermatol Online J. 15;18(9):1.

28. Van Der Geer S, Martens J, Van Roij J, Brand E, Ostertag Ju, Verhaegh Me, Neumann Ha, Krekels Ga. (2012) Imiquimod 5\% Cream As Pretreatment Of Mohs Micrographic Surgery For Nodular Basal Cell Carcinoma In The Face: A Prospective Randomized Controlled Study. $\mathrm{Br}$ J Dermatol. 67(1):110-5.

29. So Pl, Fujimoto Ma, Epstein Eh Jr. (2008) Pharmacologic Retinoid Signaling And 
Physiologic Retinoic Acid Receptor Signaling Inhibit Basal Cell Carcinoma Tumorigenesis. Mol Cancer Ther. 7(5):1275-84

30. Bianchi L, Orlandi A, Campione E, Angeloni C, Costanzo A, Spagnoli Lg, Chimenti S. (2004) Topical Treatment Of Basal Cell Carcinoma With Tazarotene: A Clinicopathological Study On A Large Series Of Cases. Br J Dermatol. 151(1):148-56 